\title{
Loss of T cell microRNA provides systemic protection against autoimmune pathology in mice
}

\author{
Lei Tian $^{\mathrm{a}}$, Gert De Hertogh ${ }^{\mathrm{b}}$, Maya Fedeli ${ }^{\mathrm{c}}$, Kim A. Staats ${ }^{\mathrm{d}, \mathrm{e}}$, Susann Schonefeldt ${ }^{\mathrm{a}}$, \\ Stephanie Humblet-Baron ${ }^{\text {a,f }}$, Ludo Van Den Bosch ${ }^{\text {d,e }}$, Paolo Dellabona ${ }^{c}$, James Dooley ${ }^{\mathrm{a}, *}$, \\ Adrian Liston $^{\mathrm{a}, *}$ \\ a Autoimmune Genetics Laboratory, VIB and University of Leuven, Leuven, Belgium \\ ${ }^{\mathrm{b}}$ Department of Morphology and Molecular Pathology, University of Leuven, Leuven, Belgium \\ ' Department of Immunology, Transplantation and Infectious Diseases, H. San Raffaele Scientific Institute, Milan, Italy \\ ${ }^{\mathrm{d}}$ Vesalius Research Center, VIB and University of Leuven, Leuven, Belgium \\ e Laboratory for Neurobiology, University of Leuven, Leuven, Belgium \\ ${ }^{\mathrm{f}}$ GIGA I3, University of Liege, Liege, Belgium
}

\section{A R T I C L E I N F O}

\section{Article history:}

Received 6 August 2011

Received in revised form

8 December 2011

Accepted 8 December 2011

\section{Keywords:}

Dicer

microRNA

T cells

Immune tolerance

Autoimmunity

\begin{abstract}
A B S T R A C T
With an increasing number of studies demonstrating alterations in T cell microRNA expression during autoimmune disease, modulation of the T cell microRNA network is considered a potential therapeutic strategy. Due to the complex and often opposing interactions of individual microRNA, prioritization of therapeutic targets first requires dissecting the dominant effects of the T cell microRNA network. Initial results utilizing a unidirectional screen suggested that the tolerogenic functions were dominant, with spontaneous colitis resulting from T cell-specific excision of Dicer. Here we performed a bidirectional screen for microRNA function by removing Dicer from the T cells of both wildtype mice and Transforming Growth Factor $\beta$ (TGF $\beta$ ) receptor-deficient mice. This allowed the impact of microRNA loss on T cell activation, effector $\mathrm{T}$ cell differentiation and autoimmune pathology to be systematically assessed. This bidirectional screen revealed a dominant immunogenic function for $\mathrm{T}$ cell microRNA, with potent suppression of T cell activation, IFN $\gamma$ production and autoimmune pathology in all targeted organs except the colon, where Dicer-dependent microRNA demonstrated a dominant tolerogenic function. These results reverse the original conclusions of microRNA function in T cells by revealing a systemic proautoimmune function.
\end{abstract}

(c) 2011 Elsevier Ltd. All rights reserved.

\section{Introduction}

The role of $\mathrm{T}$ cell microRNA ( $\mathrm{miR}$ ) in autoimmune disease has generated interest both as potential mediators of pathology, and hence putative therapeutic targets, and as biomarkers for disease. Many individual miR expressed within T cells have been linked to a variety of autoimmune diseases, including rheumatoid arthritis, systemic lupus erythematosus, multiple sclerosis, primary biliary

\footnotetext{
* Corresponding authors. Tel.: +32 163 30934/+32 163 30583(Admin)/ +32163 30590(Lab); fax: +32 016330591 .

E-mail addresses: lei.tian@student.kuleuven.be (L. Tian), gert.dehertogh@ uz.kuleuven.ac.be (G. De Hertogh), fedeli.maya@hsr.it (M. Fedeli), Kim.Staats@ vib-kuleuven.be (K.A. Staats), susann.schonefeldt@med.kuleuven.be (S. Schonefeldt) Stephanie.HumbletBaron@med.kuleuven.be (S. Humblet-Baron), ludo.vandenbosch@ vib-kuleuven.be (L. Van Den Bosch), dellabona.paolo@hsr.it (P. Dellabona), james.dooley@vib-kuleuven.be (J. Dooley), adrian.liston@vib.be (A. Liston).

URL: http://liston.vib.be
}

cirrhosis, ulcerative colitis and psoriasis [1]. As yet, however, most of these linkages remain as in vitro associations, with few systematic studies of the role of miR in autoimmune pathology.

Prediction of the potential roles of individual $\mathrm{T}$ cell $\mathrm{miR}$ in autoimmune disease largely relies on extrapolation from the biological functions determined through knockout and overexpression studies. These biological functions predict both pro- and antiautoimmune functions. Anti-autoimmune functions of $\mathrm{miR}$ include the role of miR-181 in promoting thymic negative selection [2], through the downregulation of phosphatases and subsequent increased sensitivity of TCR signaling [3]. MiR-101 and miR-184 may also be critical for peripheral $\mathrm{T}$ cell tolerance maintenance, due to the suppression of T cell activation [4,5]. Other miR may have global pro-autoimmune functions within T cells, such as miR-142$3 p$, which impedes the production of tolerogenic cAMP [6]. Altered expression of each of these miR within T cells is therefore expected to modulate autoimmune susceptibility. 
While the miR above may be expected to have relatively predictable effects on autoimmunity, based on the known biological properties, other miR are more likely to have contextual roles in autoimmunity, either due to multiple opposing functions or subsetspecific functions. As an example of the first, miR-146a has the immunogenic property of reducing the sensitivity of $\mathrm{T}$ cells to tolerance via the Fas pathway [7] and the tolerogenic property of increasing regulatory T cell suppressive capacity [8]. The net effect of enhanced autoimmunity in the miR-146a knockout mouse may indicate that the latter role is dominant over the former, or it may represent the phenotypic dominance of the function of miR-146a in the myeloid lineage [9]. Likewise, miR-155 has seemingly opposing effects, with an enhancement of regulatory T cell homeostasis [10] and also of Th17 differentiation [11]; the latter role is dominant in determining the susceptibility to Experimental Autoimmune Encephalomyelitis (EAE) [11]. Other miR are implicated in particular effector T cell lineages, such as miR-155 suppression of Th2 induction [12,13], miR-29 suppression of Th1 activity [14,15], miR-326 amplification of Th17 induction [16] and miR 17-92 suppression of Bcl-6-mediated Tfh activity [17]. The effect of altered expression of these miR are likely to be dependent on the predominant effector lineage in a specific disease, such as the ability of miR-326 overexpression to exacerbate Th17-mediated EAE [16]. With such a diverse set of individual miR, each with opposing functions, it is critical to determine the phenotypic dominance of pro- and antiautoimmune miR through a whole network approach.

The answer to this simple, yet critical, question of whether the net effect of the T cell miR transcriptome is tolerogenic or immunogenic remains unclear. Deletion of the miR network via Cremediated loss of Dicer results primarily in thymic differentiation defects, with no obvious pathological consequence [18,19]. Careful examination of CD4-Cre Dicer-floxed mice demonstrates a muted immune pathology, with colitis developing at 3-4 months of age, a phenotype associated with decreased regulatory $T$ cell numbers [20]. These results suggest a weak net positive effect of miR on T cell tolerance processes, and are in marked contrast to the rapid and fatal autoimmunity caused by regulatory $\mathrm{T}$ cell-specific depletion of the miR network [21-23]. However, as the wildtype context is that of no autoimmunity, analysis of unchallenged CD4-Cre Dicer-floxed mice constitutes a unidirectional screen, sensitized only to increases, and not decreases, in autoimmune pathology. In order to formally test the net impact of the T cell miR network using a bidirectional screen we eliminated the $\mathrm{T}$ cell miR network in both the wildtype (no challenge) context and the TGF $\beta$ receptor-deficient context of strong autoimmunity. Unlike the published unidirectional screen, this bidirectional screen found that the primary impact of the Dicerdependent miR network in T cells is to potentiate autoimmunity, as loss of miR in TGF $\beta$ receptor-deficient mice resulted in a profound reduction and delay in autoimmune pathology. These data indicate that the spontaneous colitis observed in CD4-Cre Dicer-floxed mice represents an organ-specific exception where miR has a tolerogenic function, as the phenotypically dominant function of $\mathrm{T}$ cell miR at a systemic level is immunogenic.

\section{Material and methods}

\subsection{Mice}

CD4-Cre, TGF $\beta R I I^{f l}$, and Dicer ${ }^{f l}$ mice were all backcrossed to the C57BL/6 background [24-26]. Experimental mice were agematched and housed under specific pathogen-free conditions. Cohorts of mice for the survival test were monitored for ill health and were removed from the study at death or when veterinary advice indicated likely death within $48 \mathrm{~h}$. All mice were used following ethics approval of the University of Leuven mouse facility.

\subsection{Histological examination}

Histological analysis was performed on formalin-fixed tissues using hematoxylin and eosin staining. Colitis severity was scored on a scale of $0-10$ using the accumulative score of four criteria: absence or presence of crypt abscesses (0-1) and severity of destruction of normal mucosal architecture (0-3), degree of cellular infiltration ( $0-3)$ and degree of goblet cell depletion (0-3), where 0 indicates absence, 1 indicates mild severity, 2 indicates moderate severity and 3 indicates high severity (modified from [27]). Inflammation of the lung (0-3) was scored on diffused lymphoid cell accumulation, indicating absence $(0)$, mild accumulation (1), extensive accumulation (2) and lymphoid cell aggregates (3), modified from $[28,29]$. Liver pathology was scored on a scale of 0-5 using the accumulative score of three criteria: absence or presence of fatty change (0-1), and extramedullary haematopoiesis of the portal $(0-2)$ and lobule $(0-2)$, with absence $(0)$, mild $(<50 \%$ area, 1$)$ and extensive ( $>50 \%$ area, 2 ) haematopoiesis (modified from $[30,31]$ ). Skin pathology was scored 0 , for absence of lymphoid cell infiltration, 1 , for presence of lymphoid cell infiltration, or 2, for the formation of lymphoid aggregates. Stomach pathology was scored 0 , for absence of lymphoid plasmablastic infiltration, 1, for presence of lymphoid plasmablastic infiltration within the mucosal region, or 2, for presence of lymphoid plasmablastic infiltration beyond the mucosal region. Pancreas pathology was scored 0 , for absence of lymphoid cell accumulation, 1, for presence of lymphoid cell accumulation at the periduct, or 2 , for presence of lymphoid cell accumulation in the lobule.

\subsection{Flow cytometry}

Cell surface staining and flow cytometric analysis were performed as described elsewhere [32], using anti-CD4-PE (RM4-5, eBioscience), PerCP-Cy5.5 (RPA-T4, eBioscience) or APC-Cy7 (GK1.5, BD), anti-CD8 $\alpha-$ APC or APC-eFluor780 (53-6.7 eBioscience), anti-B220-PE-Cy7 (RA-6B2, eBioscience), anti-CD19PE-Cy7 (6D5, BioLegend), anti-CD3e-APC (145-2C11, eBioscience), anti-CD44-PerCP-Cy5 (IM7, eBioscience), anti-ICOS-PE (7E.17G9, eBioscience), anti-CD62L-PE-Cy7 (MEL-14, eBioscience), antiNK1.1-PerCP-Cy5.5 (PK136, BioLegend) and TCR $\beta$-APC (H57-597, BioLegend). FITC-DEVD-FMK (Abcam) staining was performed at $37{ }^{\circ} \mathrm{C}$ for $1 \mathrm{~h}$. Intracellular staining with Ki67 (B56, BD), anti-IFN $\gamma$ APC or PE-Cy7 (XMG1.2, eBioscience), anti-IL-17A-APC (eBio17B7,eBioscience), anti-IL-4-PE-Cy7 (BVD6-24G2, eBioscience) and anti-IL-10-APC (JES5-16E3, eBioscience) antibodies was performed following fixation and permeabilization using the reagents from the eBiosciences Foxp3 staining kit. Liver leukocytes were isolated by Percoll Plus (GE Healthcare) gradient. iNKT cells were stained with PBS57 preloaded mCD1d-PE tetramers (NIH tetramer facility), or control unloaded mCD1 tetramers, following incubation with Fc block (2.4G2).

\subsection{Quantitative PCR}

Isolation of mRNA from intestine occurred by the TriPure (Roche) method and reverse transcriptase PCR with random hexamers (Life Technologies) and M-MLV (Invitrogen). Quantitative PCR was performed with the StepOnePlus (Life Technologies) with TaqMan Fast Universal PCR Master Mix (Life Technologies). The following assays were used: $\beta$-actin (4352341E, Life Technologies), polr2a (Mm00839502_m1, Life Technologies), GAPDH (Mm99999915_g1, Life Technologies), IFN $\gamma$ (Mm01168134_m1, Life Technologies), IL-4 (Mm.PT.47.16335234, IDT), IL-17a (Mm.PT.47.6531092, IDT) and IL10 (Mm.PT.45.11509489, IDT). Relative gene expression was determined by the $2^{-\Delta \Delta \mathrm{ct}}$ method and normalized to the average of the 
wildtype group. Graphs represent the relative gene expression as calculated by the $\beta$-actin expression. Determining relative expression by polr2a or GAPDH both confirmed the differences between the genotypes as detected with $\beta$-actin.

\subsection{Statistics}

Differences in survival rates were analyzed using a log rank test (Prism). All other statistical analysis was performed through an ANOVA followed by individual $t$-test comparisons, with $p<0.05$ used as the threshold for statistical significance.

\section{Results}

3.1. The T cell microRNA network is essential for a robust IFN $\gamma$ response in the absence of TGF $\beta$

T cell-specific excision of Dicer has been demonstrated to result in minor immune pathology, suggesting a weak anti-autoimmune function of the $\mathrm{T}$ cell $\mathrm{miR}$ network. In order to formally test the function of the $\mathrm{T}$ cell $\mathrm{miR}$ network during autoimmunity, we created a bidirectional screen where $T$ cell Dicer-deficiency was layered upon either the "no challenge" context (wildtype mice vs CD4-Cre Dicer fl/fl mice) or the "strong autoimmune" context (CD4Cre TGF $\beta R I I^{f / f l}$ mice vs CD4-Cre Dicer ${ }^{f / f l}$ TGF $\beta R I^{f l / f l}$ mice).

Both Dicer-deficiency and TGF $\beta$-deficiency altered basic CD4 and CD8 $\mathrm{T}$ cell numbers, with Dicer-deficiency resulting in decreases in CD4 and CD8 peripheral numbers, and TGF $\beta$-deficiency resulting in an expansion of both populations within the spleen (Supplementary Fig. 1). Beyond the developmental defects altering $\mathrm{T}$ cell numbers, profound differences were observed in $\mathrm{T}$ cell activation. The effect of Dicer-deficiency in the "no challenge" context was a weak reduction in T cell activation, with no change in ICOS expression on CD4 T cells from the lymph nodes (Fig. 1a,b) and a slight reduction in the spleen (Supplementary Fig. 2).
Proliferation and apoptosis of CD4 T cells remained relatively normal (Fig. 1c,d, Supplementary Fig. 2). When Dicer-deficient mice were aged beyond 13 weeks, the defect in activation was partially mitigated, with a minor increase in CD4 T cell activation in the lymph nodes, data in line with the previous observation that, over time, immune dysregulation develops in Dicer-deficient mice [20]. The effect of Dicer-deficiency in the reverse screen, the "strong autoimmune" context, was a substantial 10-fold decrease in CD4 T cell activation. TGF $\beta$ RII-deficient mice showed a strong increase in T cell activation, with ICOS expressed on $\sim 50 \%$ of CD4 T cells (Fig. 1b) and with $65 \%$ of cells undergoing proliferation (Fig. 1c). When Dicer-deficiency was layered upon TGF $\beta$ RII-deficiency, upregulation of ICOS was reduced to $\sim 5 \%$ and proliferation rates returned to normal, representing a profound defect in T cell activation. Similar results were observed in the spleen (Supplementary Fig. 2) and for alternative $\mathrm{T}$ cell activation markers CD44 and CD62L (Supplementary Fig. 3). Apoptosis rates were increased in TGFßRIIdeficient mice, but no consistent effect of adding Dicer-deficiency was observed (Fig. 1d, Supplementary Fig. 2).

A similar effect was observed for CD8 T cells, where Dicerdeficiency in the "no challenge" context resulted in a slight reduction in ICOS expression (Fig. 2a,b) and little change in proliferation rates (Fig. 2c). In contrast to CD4 T cells, Dicer-deficiency increased the apoptosis rate in CD8 T cells (Fig. 2d). The effect of Dicer-deficiency in the reverse screen, the "strong autoimmune" context, was a strong defect in CD8 T cell activation, with a 10-fold reduction in ICOS expression (Fig. 2b) and a returning of proliferation rates to baseline levels (Fig. 2c). TGFßRII-deficiency caused an increase in $\mathrm{CD} 8$ apoptosis rates, however these were not synergistic with the apoptotic phenotype of Dicer-deficiency (Fig. 2d). Similar results were observed in the spleen (Supplementary Fig. 4) and for alternative $\mathrm{T}$ cell activation markers CD44 and CD62L (Supplementary Fig. 3). These results demonstrate that the net effect of Dicer-deficiency in both CD4 and CD8 T cells is a mild suppression of T cell activation, in the young "no challenge" context a

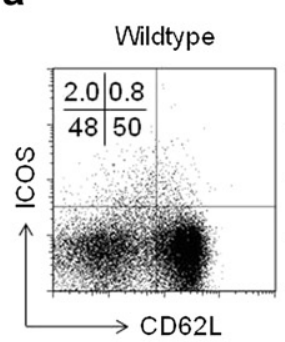

Dicer-flox

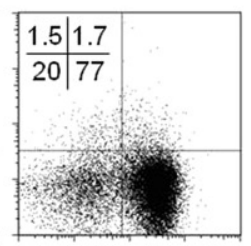

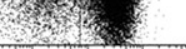

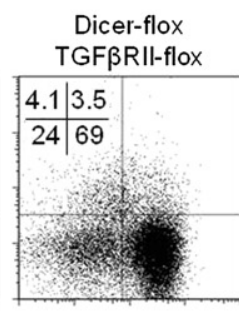

b

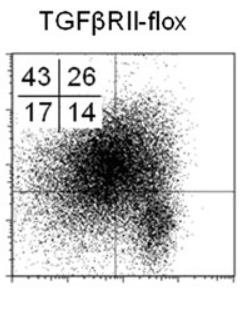

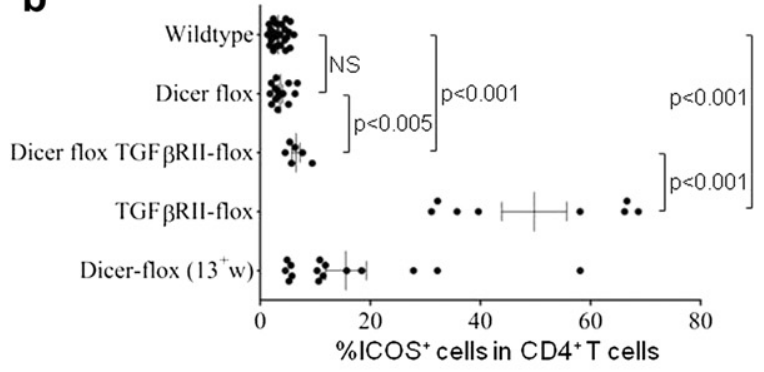

C

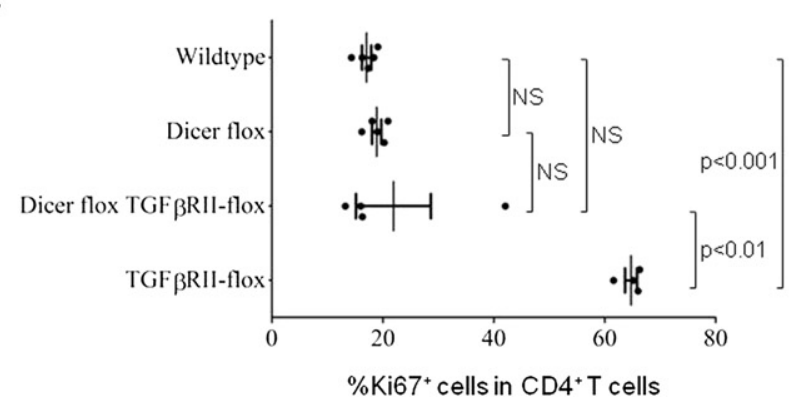

d

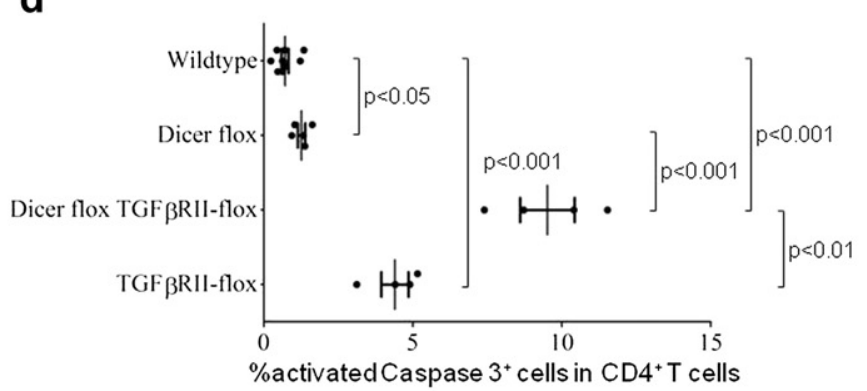

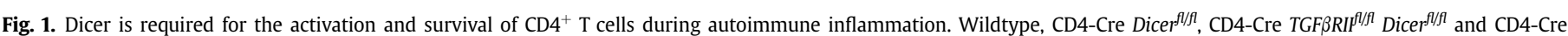

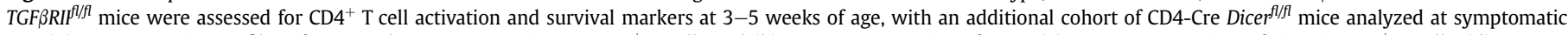

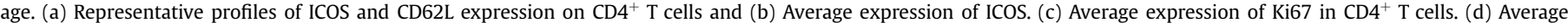

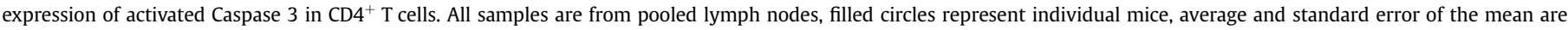
indicated. 

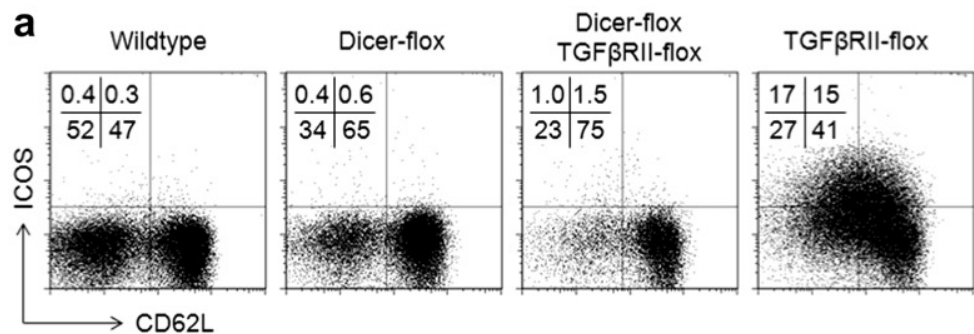

b

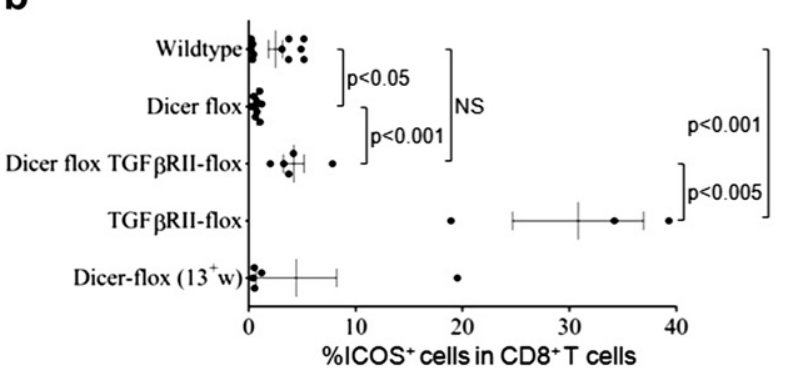

C

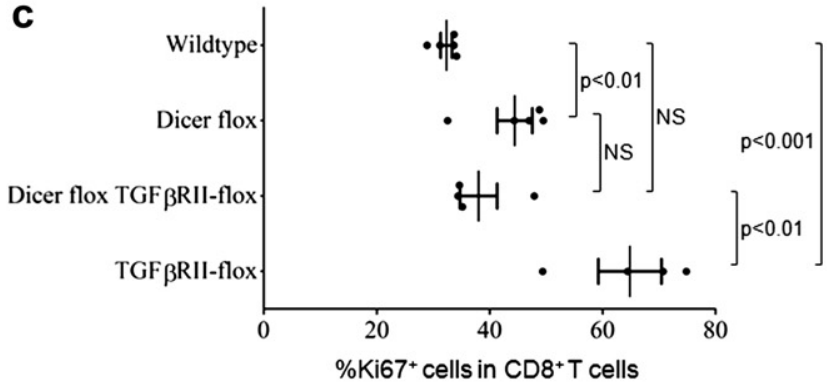

d

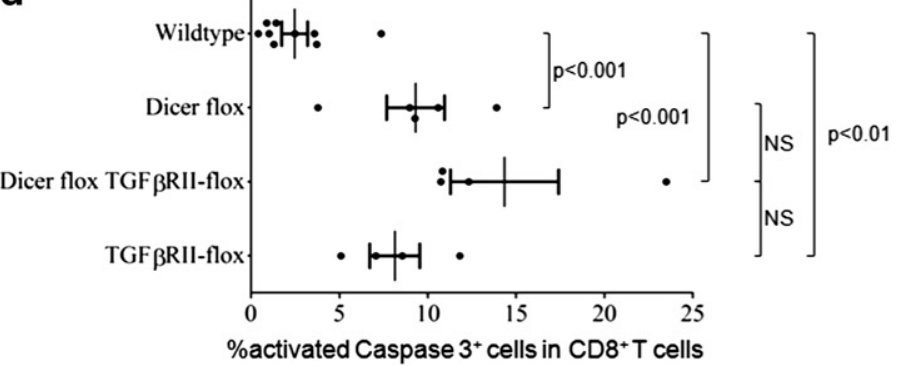

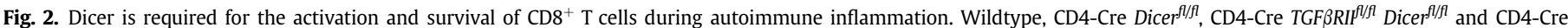

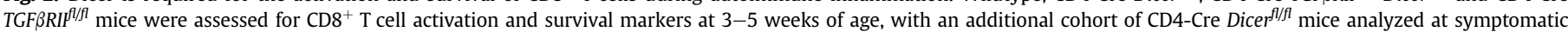

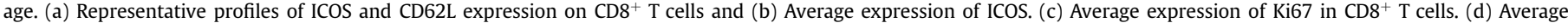

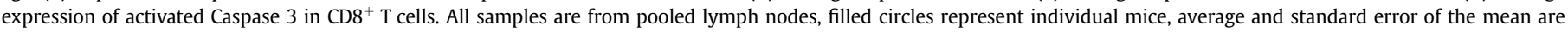
indicated.

which is revealed to be a strong suppression of activation when challenged by the "strong autoimmune" context.

Individual miR have been demonstrated to have both positive and negative regulatory influences over entry into effector $T$ cell subsets. To determine whether the broad $\mathrm{T}$ cell activation phenotype encompassed shifts in the relative generation of T cell subsets, we measured the effect of Dicer-deficiency on T cell production of IFN $\gamma$, IL-4, IL-17 and IL-10. In the "no challenge" context, minimal production of any of these cytokines by CD4 T cells was observed (Fig. 3 and Supplementary Fig. 5), and the only effect observed by the addition of Dicer-deficiency was a mild decrease in IFN $\gamma$ production in the spleen (Supplementary Fig. 5a,b). As with general $\mathrm{T}$ cell activation, this phenotype was partially mitigated with age, when Dicer-deficient mice developed increased numbers of Th1 and Th17 cells (Fig. 3b,f and Supplementary Fig. 5b,f). A similar result was observed with IFN $\gamma$ production by $\mathrm{CD} 8 \mathrm{~T}$ cells, with initial suppression by Dicer-deficiency followed by slight overproduction with age (Fig. 4and Supplementary Fig. 6). As with the general activation profile, these results demonstrate that Dicerdeficiency does not drive spontaneous effector T cell differentiation from a young age, with the primary exception being an increase in Th17 cells in older mice.

The "strong autoimmune" context of TGF $\beta$ RII-deficiency creates a primarily Th1-drive immune response, with $\sim 40 \%$ of CD4 and CD8 T cells expressing IFN $\gamma$ in both the lymph nodes (Figs. 3b and 4b) and spleen (Supplementary Fig. 5b, Supplementary Fig. 6b). No increase in Th17 immunity and only minor increases in Th2 immunity are observed (Fig. 3 and Supplementary Fig. 5). Previous models postulating a net protective role for miR in preventing autoimmunity predict that laying Dicer-deficiency onto TGF $\beta$ RIIdeficiency would result in increased cytokine production. By contrast, dual deficiency led to a 10 -fold reduction in Th1 responses (Fig. 3b and Supplementary Fig. 5) and a four-fold reduction in IFN $\gamma$ production by CD8 T cells when compared to TGF $\beta$ RII-deficiency alone (Fig. 4b and Supplementary Fig. 6b). No difference was observed in CD4 T cell production of IL- 4 or IL-10, however dual deficient mice showed a significant increase in Th17 (Fig. 3 and Supplementary Fig. 5). These results demonstrate that the miR network has a pro-Th1 and anti-Th17 effect on T cells, resulting in a diminishment of Th1 responses and an expansion of Th17 responses upon Dicer excision.

\subsection{A bidirectional screen on Dicer-deficiency reveals that miR in $T$ cells drive pathology in the colon but impede autoimmunity in other anatomical locations}

The essential role of miR in enabling Th1 expansion in the absence of TGF $\beta$ signaling indicates the potential for loss of the miR network to quench the resulting autoimmune pathology. In order to test for such a function we aged a cohort of wildtype and CD4-Cre $T G F \beta R I I^{f / f l}$ mice, with and without the excision of Dicer, resulting in mice of four different genotypes - wildtype, CD4-Cre Dicer ${ }^{f / f l}$ mice,

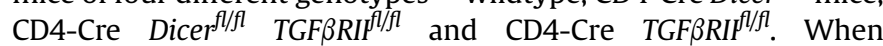
compared to wildtype mice, the immune dysregulation observed in older CD4-Cre Dicer fl/fl mice manifested in a slight increase in mortality, with $\sim 15 \%$ of mice dying between 13 and 17 weeks of age (Fig. 6a). As previously demonstrated [33], loss of TGFßRII resulted in $100 \%$ fatal autoimmunity at 3-4 weeks of age (Fig. 6a). When Dicer-deficiency was combined with TGF $\beta$ RII-deficiency fatal autoimmunity was still observed in $100 \%$ of mice, however the disease was significantly delayed, with death occurring between 9 and 17 weeks of age (Fig. 6a).

The effect of Dicer-deficiency on disease progression was mirrored by changes in organ pathology. To assess pathology during the period of fatal illness, a histological review was conducted at 9-17 weeks for wildtype, CD4-Cre Dicer ${ }^{f / f l}$ mice and CD4Cre Dicer fl/fl TGF $R$ RIf $^{f l / f l}$ mice, the period of fatality for Dicer- and dual deficient mice, at 3-4 weeks for CD4-Cre TGFßRII fl/fl mice, the equivalent point in this more severe disease course. As previously recognized, Dicer-deficiency increased the incidence of inflammation of the colon, with lesions observed in $>80 \%$ of mice (Figs. $6 \mathrm{~b}$ and 7), manifesting as submucosal infiltration, crypt abscesses, 
a

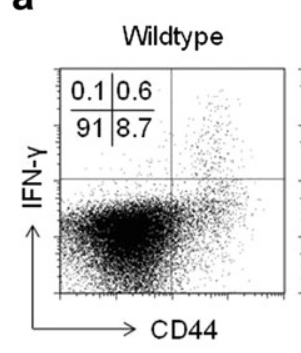

C

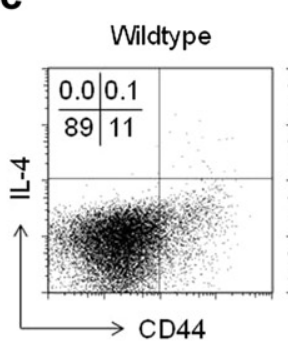

e

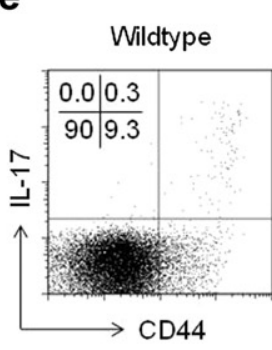

g

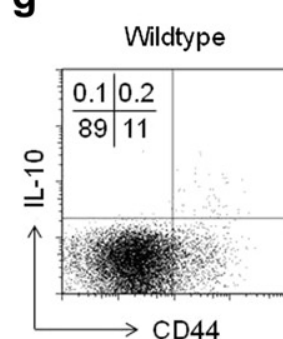

Dicer-flox
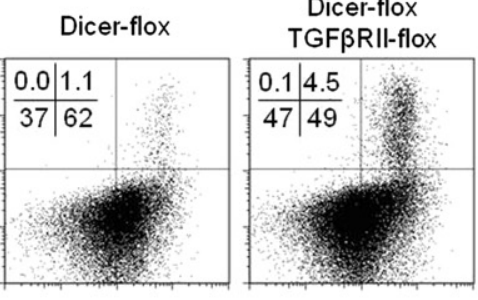

TGFßRII-flox

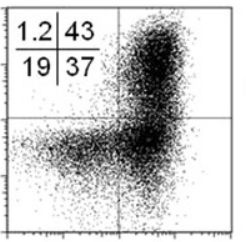

b

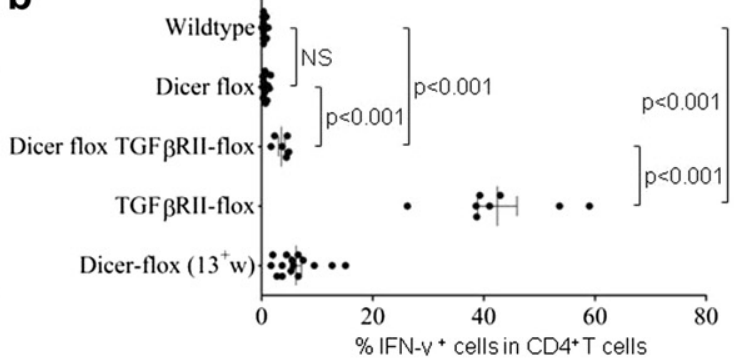

d
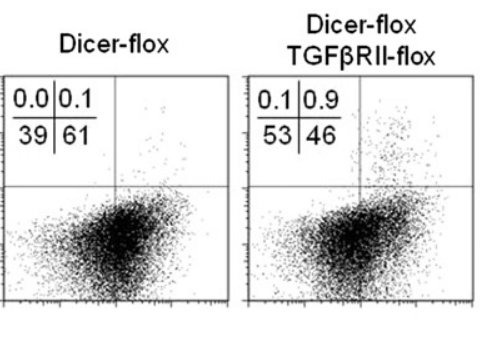

TGF $\beta$ RII-flox
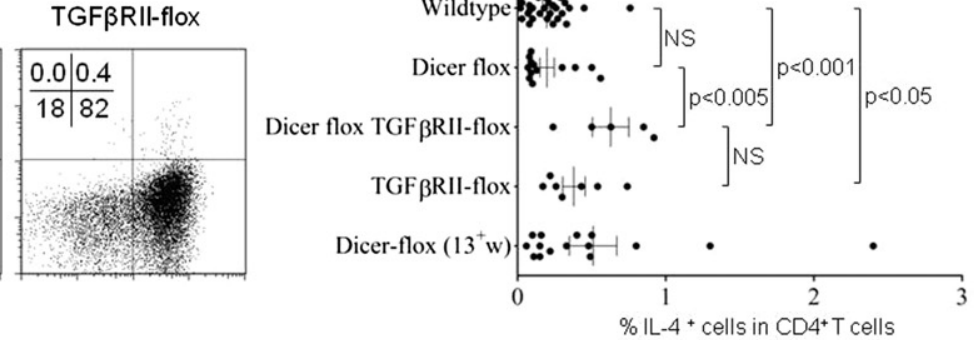

f
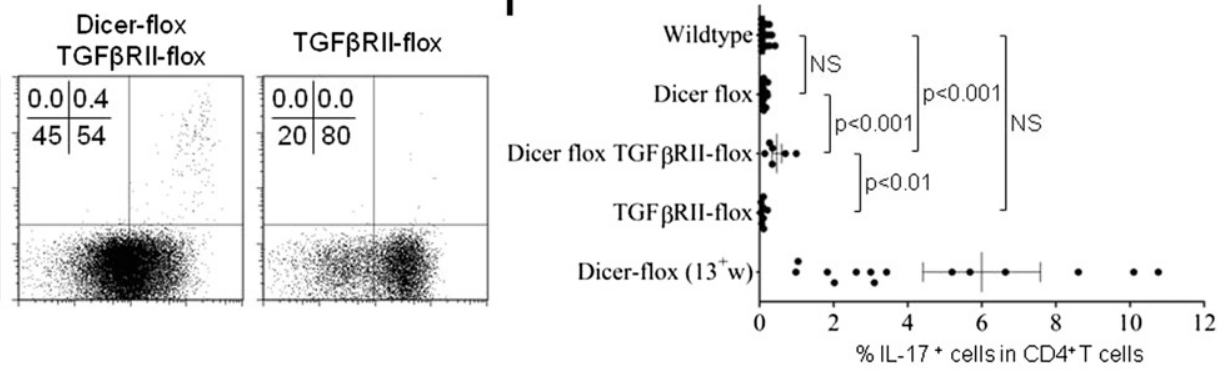

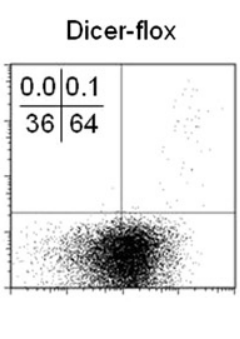

h
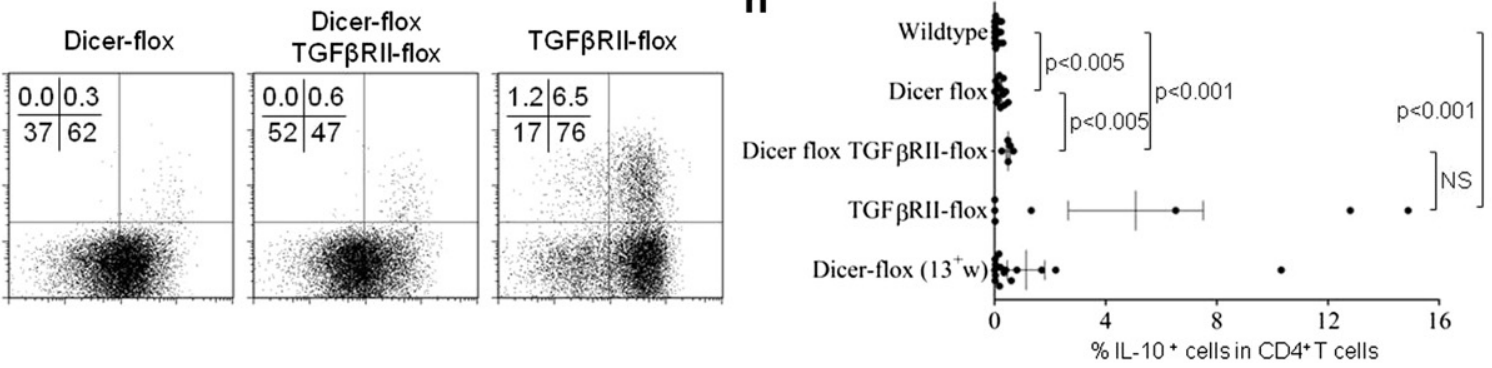

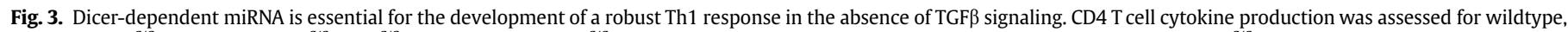

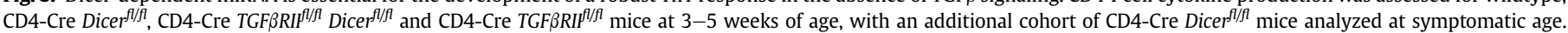

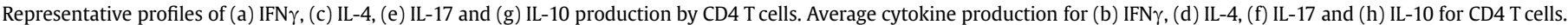
All samples are from pooled lymph nodes, filled circles represent individual mice, average and standard error of the mean are indicated.

ulceration and goblet cell loss. Clear lesions in the liver were also identified in one of twelve mice, but no inflammation was observed in the lung, skin, pancreas, heart, stomach, small bowel or kidney (Figs. 6b and 7). The inflammation of the liver observed was likely secondary to colitis, with only light cell infiltration at the portal tract, however it could have been due to the loss of the regulatory effect of NKT cells, which are profoundly reduced in number (Supplementary Fig. 7), in light with our previous observation [34]. In the "strong autoimmunity" context, 100\% of CD4-Cre TGFRRIf fl/fl mice developed inflammation in the lung, liver, pancreas, heart, stomach and kidney. Inflammation was also common in the skin and colon, while the small bowel remained unaffected (Figs. 6b and 7). Dual deficient mice, with defects in both TGF $\beta$-mediated tolerance and miR production, demonstrated a sharp reduction in the scope of pathology, with only a minority of mice developing inflammation of the lung, liver or stomach, and no mice developing inflammation of the skin, pancreas, heart or kidney (Figs. 6b and 7). In TGF $\beta$ RII-deficient mice lung pathology was the likely cause of death, with severe alveolitis, signs of bleeding and diffuse interstitial lung disease; manifestations which were far more severe than the mild disease noted in the sole dual deficient mouse which manifested lung pathology (Fig. 7). Like both the Dicer-deficient and TGFßRII-deficient mice, dual deficient mice developed colitis with high frequency. The reduction in the incidence of immune pathology in dual deficient mice compared to TGFßRII-deficient mice was accompanied by a reduction in the severity of pathology, with the exception of the colon, where severity of pathology was comparable across the genotypes (Figs. 5 and 6). Analysis of 
a

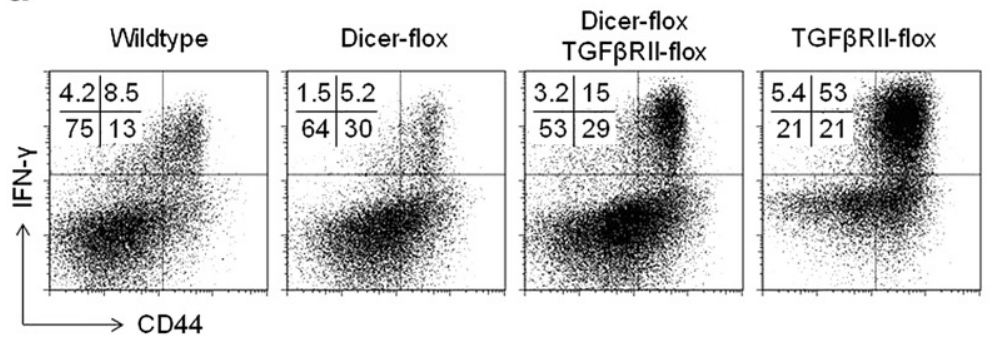

b

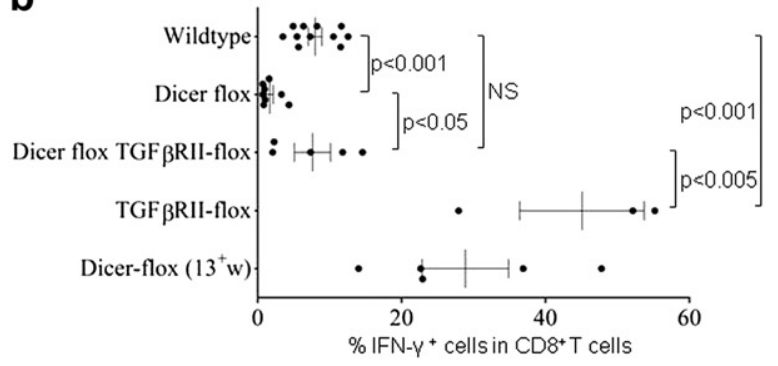

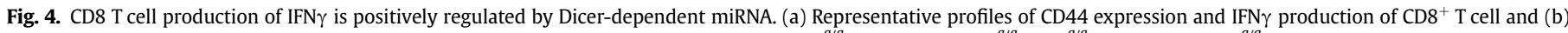

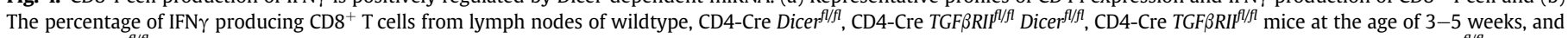

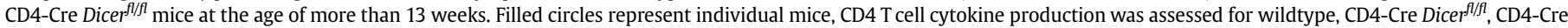
TGF RRII ${ }^{f / f l}$ Dicer $^{f l / f l}$ and CD4-Cre TGF RRIP/flf mice at 3-5 weeks of age, with an additional cohort of CD4-Cre Dicer ${ }^{f / / f l}$ mice analyzed at symptomatic age.

cytokine expression in the colon found the primary shifts in expression were a substantial increase in IFN $\gamma$ and a significant increase in IL-10 in TGFßRII-deficient mice, changes which were not observed in Dicer-deficient or double-deficient mice (Supplementary Fig. 8). These data suggest that the increase in autoimmunity in the colon of Dicer-deficient mice is the exception rather than the rule, with the susceptibility to autoimmune pathology in all other organs surveyed being reduced by the loss of miR.

\section{Discussion}

Understanding the global impact of the miR network in T cells is the critical starting point for investigations into the function of
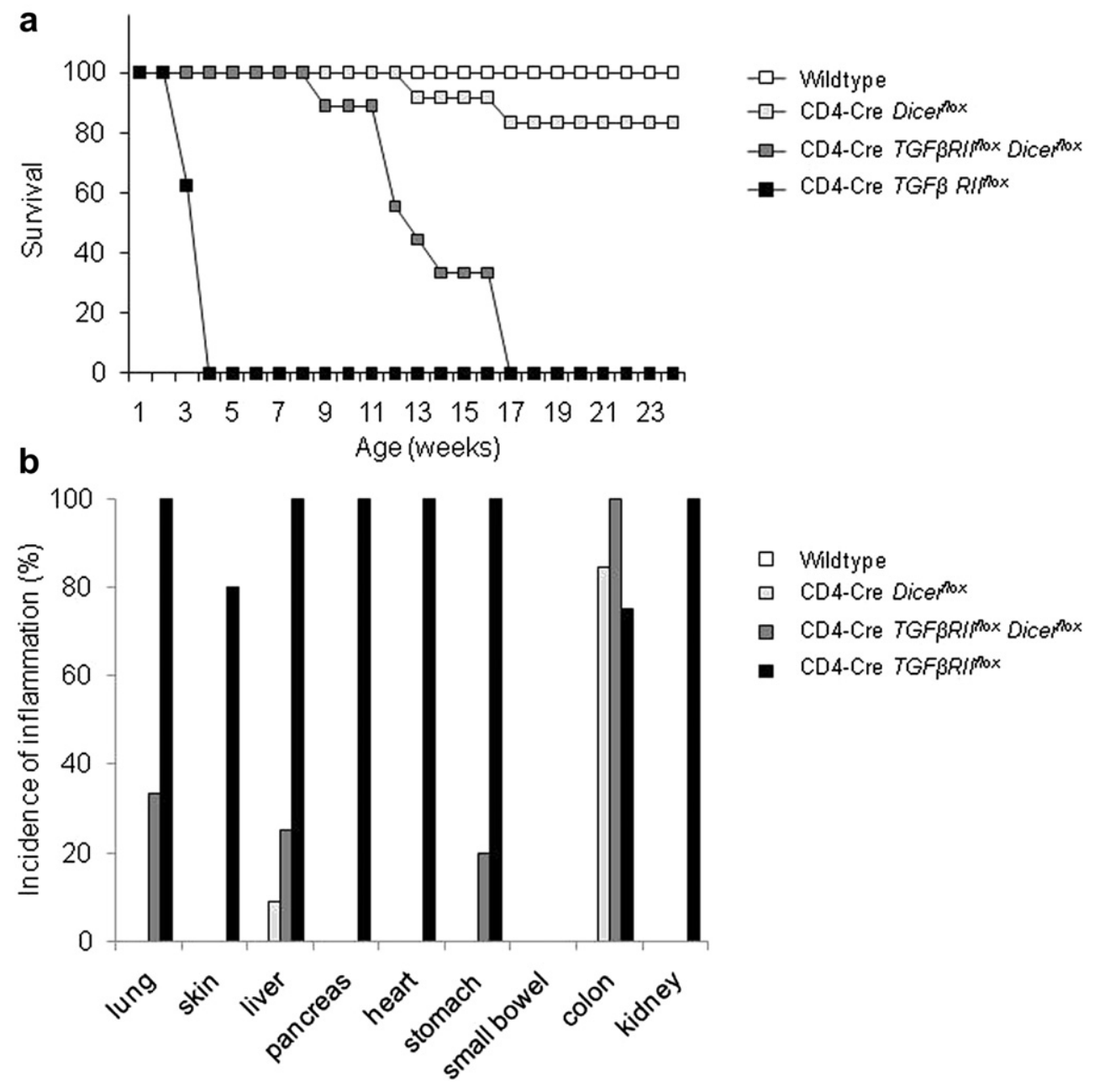

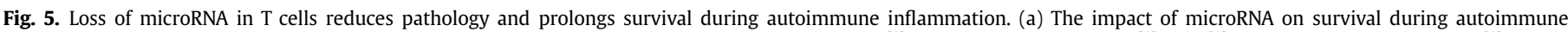

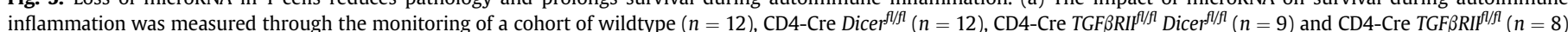

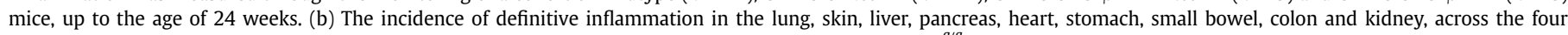
genotypes, as measured through histological examination at 13-17 weeks of age (except CD4-Cre TGFRRIf/fl mice, assessed at 3-4 weeks of age). 


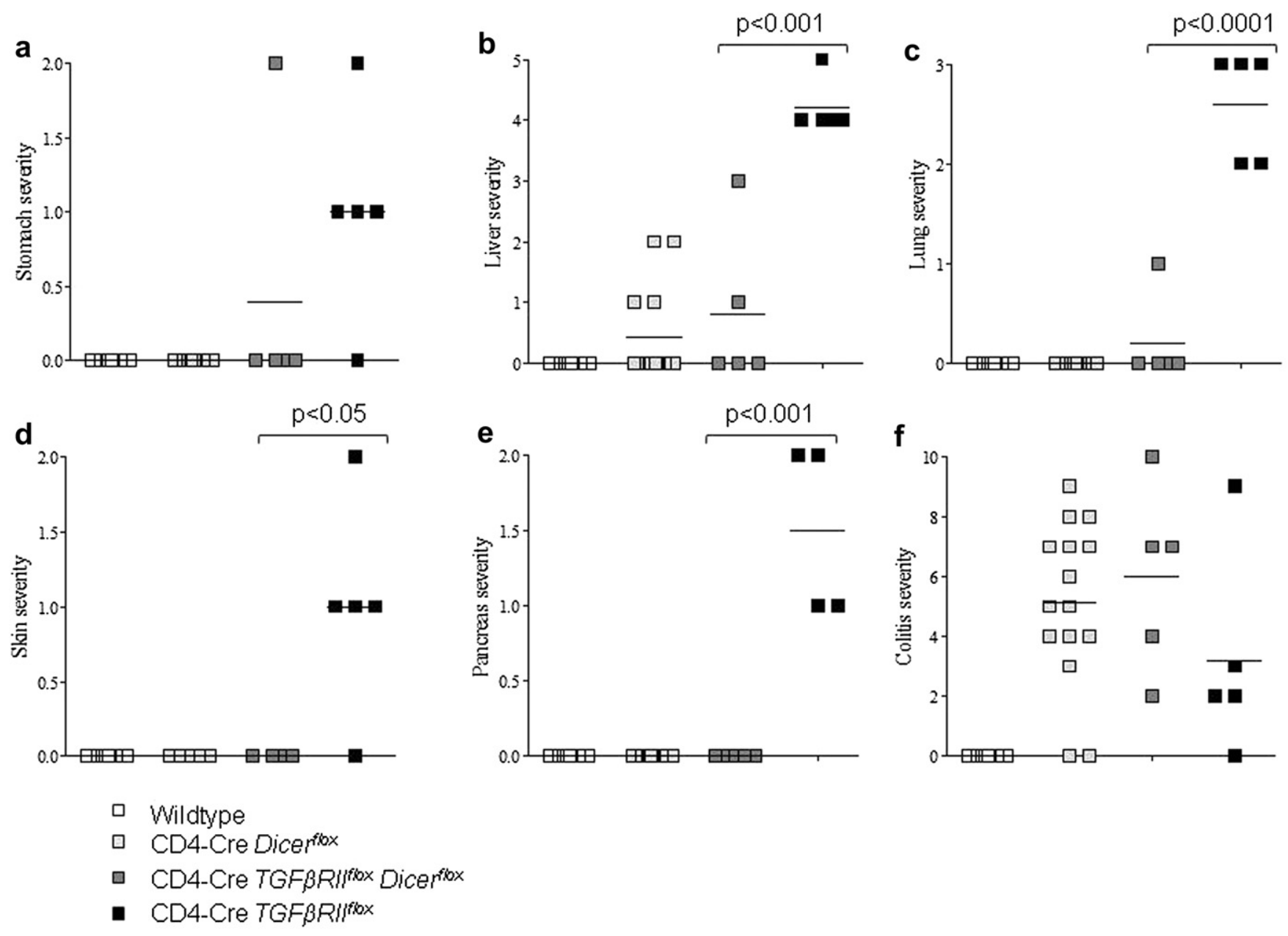

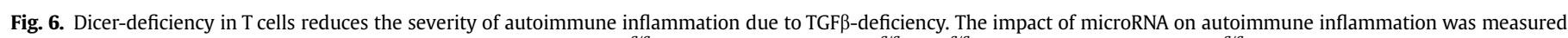

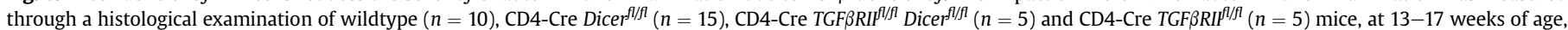

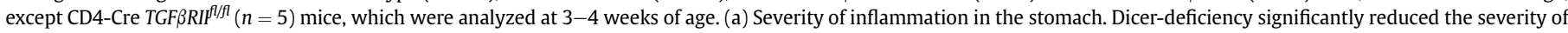

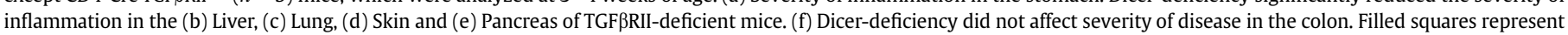
individual mice of each genotype.

individual miR:mRNA interactions. Previous studies on the effect of the miR network in T cells have either utilized in vitro approaches or have evaluated the effect of Dicer excision in a "no challenge" in vivo context. The use of a unidirectional "no challenge" screen only allows heightened susceptibility to autoimmunity to be detected; employing a bidirectional screen is required to present a balanced test of miR function. Here we created such a bidirectional screen by layering Dicer-deficiency on either the wildtype "no challenge" state or the TGF $\beta$ RII-deficient "strong autoimmunity" condition, allowing us to assess the net contribution that Dicer-dependent miR make to $\mathrm{T}$ cell activation, polarization and autoimmune function. The results of the in vivo bidirectional screen differ strongly from both the previously published unidirectional screen and in vitro experiments on important aspects such as the role of miR on $\mathrm{T}$ cell polarization and autoimmunity.

Previous data on the function of miR on T cell subsets was largely limited to in vitro polarization experiments, where T cells lacking Dicer exhibited preferential Th1 induction under non-polarizing conditions [19], while Th17 induction was significantly impaired [20]. The in vivo results of this screen contrast strongly with this in vitro data. In vivo we observed a profound suppression of the Th1 response, with the loss of Dicer reducing by ten-fold the spontaneous Th1 autoimmunity caused by TGF $\beta$ RII-deficiency. Likewise, while Th17 production in young Dicer-deficient mice was negligible, when immune dysregulation was introduced to the system by aging or by TGFßRII-deficiency the magnitude of the Th17 response was significantly increased by the loss of miR. Thus for both Th1 and Th17 responses, the effect of miR revealed here in vivo is the opposite of that previously postulated from in vitro experiments. The opposite polarity of the results may represent the complex counter-balancing that can exist within the miR network, with different miR amplifying or suppressing the same fate lineage by modulating different mediator molecules. In the artificial in vitro system the non-physiological balance of the importance of different polarization pathways may therefore result in an outcome opposite to that occurring in the physiological in vivo context described here.

With regards to the influence of Dicer on T cell-driven pathology, we validate here the initial observations of the unidirectional screen [20] that miR have a pro-immune regulation impact on the colon, with spontaneous colitis being observed in the Dicerdeficient background. Nevertheless, these results caution against this observation being taken as a general phenomenon, as screening for added autoimmunity on a non-autoimmune context creates a detection bias, where only increased susceptibility and not decreased susceptibility can be detected. Indeed, when we reversed the experiment by looking at the effect of Dicer-deficiency on strong autoimmunity we observed that the spontaneous colitis 


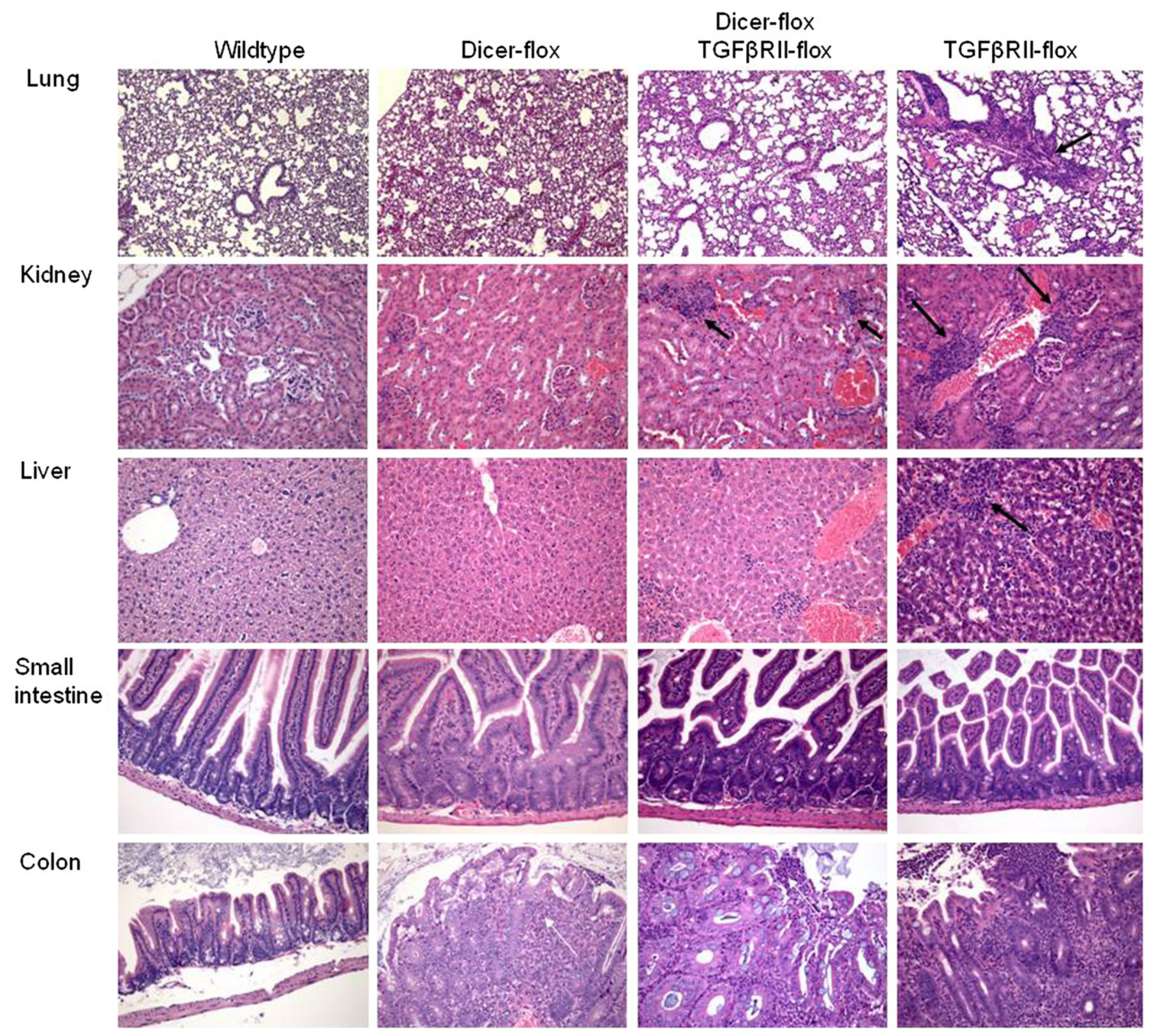

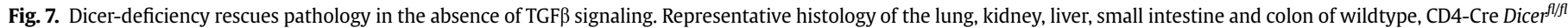

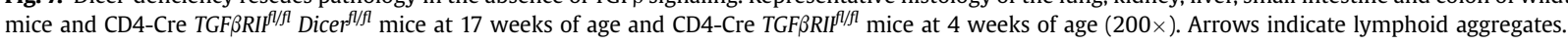

was the exception rather than the rule. Of the eight target organs affected by TGF $\beta$ signaling deficiency, the pathology of seven organs was either fully or partially rescued by additional loss of Dicer, with the colon being the sole exception. The rationale by which the colon presents with a unique phenotype no observed in other organs is open to speculation. One possibility is that T cell tolerance within the colon is dependent on regulatory $\mathrm{T}$ cell suppression to a higher extent than that of other organs, as supported by the well-established observations that deficiency in regulatory $\mathrm{T}$ cells generated through day three thymectomy or the transfer of naive $\mathrm{T}$ cells to immunodeficient mice has colitis as the primary presentation [35-37]. As loss of miR reduces both the immunogenic capacity of effector T cells (this study) and the tolerogenic capacity of regulatory $T$ cells [23], an organ-specific change in the balance of power between these two subsets could result in a reversal of the systemic phenotype. Alternatively, deficiency in Dicer increasing Th17 production while reducing IL-10 production in the colon. As IL-10 signaling is critical to prevent Th17-mediated autoimmunity in the colon [38], this cytokine imbalance may be sufficient to drive local autoimmunity despite the defects in $\mathrm{T}$ cell activation and proliferation. In other organs, where IL-10 signaling to Th17 cells is less critical for tolerance, the defects in T cell activation and proliferation outweigh this effect, creating a net increase in tolerance in Dicer-deficient mice. These results present a more comprehensive evaluation of the miR network in T cell regulation, with a tolerogenic function in protecting against Th17-associated pathology, such as in the colon, but a strong immunogenic function in driving Th1-associated pathology against a wide spectrum of target organs.

The general immunogenic function of the $\mathrm{T}$ cell miR network observed here, with a profound defect in systemic T cell activation in Dicer-deficient mice, provides a plausible explanation for the discrepancy in pathology development due to CD4-Cre and Foxp3 $3^{\text {Cre }}$ Dicer excision. The development of colitis at 3-4 months of age in 
CD4-Cre Dicer ${ }^{\text {fl/fl }}$ mice was interpreted to mean that the Dicerdependent miR network was tolerogenic in nature, with excessive T cell activation occurring in its absence [20]. However the severity of disease that results from pan-T cell excision is far lower than the disease which results from excision of Dicer specifically within the Foxp $3^{+}$subpopulation, which is typically fatal at $3-4$ weeks [21-23]. A simple subtractive analysis suggests that while the miR network is tolerogenic within Foxp ${ }^{+}$regulatory T cells, maintaining lineage integrity [23], the lower level of pathology in CD4-Cre Dicer ${ }^{f l / f l}$ mice necessitates an overall immunogenic function of Dicer-dependent $\mathrm{miR}$ in non-regulatory $\mathrm{T}$ cells. While discordant with previous in vitro experimental data, this biological model is validated by the current in vivo data, with a strong suppression of $\mathrm{T}$ cell activation in Dicer-deficient mice challenged by TGF $\beta$ RII insufficiency. In essence, the profound immunogenic defect in effector T cell activation in CD4Cre Dicer ${ }^{f / f l}$ mice can be thought of as masking the major tolerogenic defect in regulatory T cells, creating a relatively mild net phenotype. These two strong opposing phenotypes are only revealed through specific Dicer excision in Foxp3 ${ }^{\text {Cre }}$ Dicer $^{f / f l}$ mice [21-23] and through exposing CD4-Cre Dicer ${ }^{f / f l}$ mice to a strong autoimmune challenge, as in the current study.

The results presented here have important implications for the potential exploitation of $\mathrm{miR}$ as a therapeutic in autoimmune disease. An increasing amount of miR biomarker data is being generated for autoimmune disease, with studies identifying abnormalities in peripheral blood miR expression in systemic lupus erythematosus [39], rheumatoid arthritis [40] and multiple sclerosis [16], among other diseases. While functional studies will reveal an intricate web of individual miR with often opposing functions within T cells, these results suggest that searching for the strongly immunogenic miR may provide fruitful targets for therapeutic intervention, as the immunogenic miR are phenotypically dominant over the tolerogenic miR. As loss of the entire miR network resulted in a profound reduction in autoimmune pathology in the current study, targeted identification and subsequent antagonism of the specific miR required for strong autoimmunity may successfully modulate autoimmunity. At a broader level, targeted inhibition of the entire miR network, through downregulation of Dicer or related biogenesis proteins, would be predicted to reduce the severity of autoimmune pathology. Finally, the intriguing possibility exists that both positive and negative feedback loops are generated during autoimmunity through modulation of Dicer itself, as the exposure to IFN $\gamma$ increases Dicer expression [41], which has the potential to amplify Th1 responses, while exposure to IFN $\alpha$ decreases Dicer expression [41], leading to the possibility that local inflammatory events will feedback on T cell activation via modulation of the miR network.

\section{Acknowledgments}

We thank A. Tarakhovsky for providing Dicer-floxed mice and A. Rudensky for the provision of TGFRRII-floxed mice. The authors would like to thank the NIH Tetramer Core Facility for mouse CD1d tetramer. This work was supported by grants from the VIB and FWO (A.L.). A.L. is a Marie Curie Reintegration Grant Fellow and ERC Start Grant holder. L.T., G.D.H., M.F., K.S., S.S. and S.H-B performed the experiments, L.V.D.B and P.D provided experimental advice and supplied reagents, A.L. and J.D. designed the experiments and wrote the manuscript. The authors declare they have no conflicting financial interest. The spouse of A.L. is employed by UCB Pharma.

\section{Appendix. Supplementary material}

Supplementary data related to this article can be found online at doi:10.1016/j.jaut.2011.12.005.

\section{References}

[1] Furer V, Greenberg JD, Attur M, Abramson SB, Pillinger MH. The role of microRNA in rheumatoid arthritis and other autoimmune diseases. Clin Immunol 2010;136;1-15.

[2] Ebert PJ, Jiang S, Xie J, Li QJ, Davis MM. An endogenous positively selecting peptide enhances mature $\mathrm{T}$ cell responses and becomes an autoantigen in the absence of microRNA miR-181a. Nat Immunol 2009; 10:1162-9.

[3] Li QJ, Chau J, Ebert PJ, Sylvester G, Min H, Liu G, et al. miR-181a is an intrinsic modulator of T cell sensitivity and selection. Cell 2007:129:147-61.

[4] Yu D, Tan AH, Hu X, Athanasopoulos V, Simpson N, Silva DG, et al. Roquin represses autoimmunity by limiting inducible T-cell co-stimulator messenger RNA. Nature 2007:450:299-303.

[5] Weitzel RP, Lesniewski ML, Haviernik P, Kadereit S, Leahy P, Greco NJ, et al. microRNA 184 regulates expression of NFAT1 in umbilical cord blood CD4+ T cells. Blood 2009;113:6648-57.

[6] Huang B, Zhao J, Lei Z, Shen S, Li D, Shen GX, et al. miR-142-3p restricts cAMP production in CD4+CD25- T cells and CD4+CD25+ TREG cells by targeting AC9 mRNA. EMBO Rep 2009;10:180-5.

7] Curtale G, Citarella F, Carissimi C, Goldoni M, Carucci N, Fulci V, et al An emerging player in the adaptive immune response: microRNA-146a is a modulator of IL-2 expression and activation-induced cell death in T lymphocytes. Blood 2010;115:265-73.

[8] Lu LF, Boldin MP, Chaudhry A, Lin LL, Taganov KD, Hanada T, et al. Function of miR-146a in controlling Treg cell-mediated regulation of Th1 responses. Cell 2010;142:914-29.

[9] Boldin MP, Taganov KD, Rao DS, Yang L, Zhao JL, Kalwani M, et al. miR-146a is a significant brake on autoimmunity, myeloproliferation, and cancer in mice. J Exp Med 2011;208:1189-201.

[10] Lu LF, Thai TH, Calado DP, Chaudhry A, Kubo M, Tanaka K, et al. Foxp3dependent microRNA155 confers competitive fitness to regulatory $\mathrm{T}$ cells by targeting SOCS1 protein. Immunity 2009;30:80-91.

[11] O'Connell RM, Kahn D, Gibson WS, Round JL, Scholz RL, Chaudhuri AA, et al. MicroRNA-155 promotes autoimmune inflammation by enhancing inflammatory T cell development. Immunity 2010;33:607-19.

[12] Thai TH, Calado DP, Casola S, Ansel KM, Xiao C, Xue Y, et al. Regulation of the germinal center response by microRNA-155. Science 2007;316: 604-8.

[13] Rodriguez A, Vigorito E, Clare S, Warren MV, Couttet P, Soond DR, et al. Requirement of bic/microRNA-155 for normal immune function. Science 2007;316:608-11.

[14] Steiner DF, Thomas MF, Hu JK, Yang Z, Babiarz JE, Allen CD, et al. MicroRNA-29 regulates $\mathrm{T}$-box transcription factors and interferon-gamma production in helper T cells. Immunity 2011;35:169-81.

[15] Ma F, Xu S, Liu X, Zhang Q, Xu X, Liu M, et al. The microRNA miR-29 controls innate and adaptive immune responses to intracellular bacterial infection by targeting interferon-gamma. Nat Immunol 2011;12:861-9.

[16] Du C, Liu C, Kang J, Zhao G, Ye Z, Huang S, et al. MicroRNA miR-326 regulates $\mathrm{TH}-17$ differentiation and is associated with the pathogenesis of multiple sclerosis. Nat Immunol 2009;10:1252-9.

[17] Yu D, Rao S, Tsai LM, Lee SK, He Y, Sutcliffe EL, et al. The transcriptional repressor Bcl-6 directs T follicular helper cell lineage commitment. Immunity 2009;31:457-68.

[18] Cobb BS, Nesterova TB, Thompson E, Hertweck A, O'Connor E, Godwin J, et al. $\mathrm{T}$ cell lineage choice and differentiation in the absence of the RNase III enzyme dicer. J Exp Med 2005;201:1367-73.

[19] Muljo SA, Ansel KM, Kanellopoulou C, Livingston DM, Rao A, Rajewsky K. Aberrant T cell differentiation in the absence of dicer. J Exp Med 2005;202: 261-9.

[20] Cobb BS, Hertweck A, Smith J, O'Connor E, Graf D, Cook T, et al. A role for dicer in immune regulation. J Exp Med 2006;203:2519-27.

[21] Chong MM, Rasmussen JP, Rudensky AY, Littman DR. The RNAseIII enzyme Drosha is critical in T cells for preventing lethal inflammatory disease. J Exp Med 2008;205:2005-17.

[22] Zhou X, Jeker LT, Fife BT, Zhu S, Anderson MS, McManus MT, et al. Selective miRNA disruption in $\mathrm{T}$ reg cells leads to uncontrolled autoimmunity. J Exp Med 2008;205:1983-91.

[23] Liston A, Lu LF, O’Carroll D, Tarakhovsky A, Rudensky AY. Dicer-dependent microRNA pathway safeguards regulatory T cell function. J Exp Med 2008; 205:1993-2004

[24] Wolfer A, Bakker T, Wilson A, Nicolas M, Ioannidis V, Littman DR, et al. Inactivation of Notch 1 in immature thymocytes does not perturb CD4 or CD8T cell development. Nat Immunol 2001;2:235-41.

[25] Yi R, O'Carroll D, Pasolli HA, Zhang Z, Dietrich FS, Tarakhovsky A, et al. Morphogenesis in skin is governed by discrete sets of differentially expressed microRNAs. Nat Genet 2006;38:356-62.

[26] Leveen P, Carlsen M, Makowska A, Oddsson S, Larsson J, Goumans MJ, et al. TGF-beta type II receptor-deficient thymocytes develop normally but demonstrate increased CD8+ proliferation in vivo. Blood 2005;106: 4234-40.

[27] Shen C, Bullens D, Kasran A, Maerten P, Boon L, Aerts JM, et al. Inhibition of glycolipid biosynthesis by $\mathrm{N}$-(5-adamantane-1-yl-methoxy-pentyl)-deoxynojirimycin protects against the inflammatory response in hapten-induced colitis. Int Immunopharmacol 2004;4:939-51. 
[28] Gamsu G, Salmon CJ, Warnock ML, Blanc PD. CT quantification of interstitial fibrosis in patients with asbestosis: a comparison of two methods. AJR Am J Roentgenol 1995;164:63-8.

[29] Takada K, Takiguchi M, Konno A, Inaba M. Spontaneous development of multiple glandular and extraglandular lesions in aged IQI/Jic mice: a model for primary Sjogren's syndrome. Rheumatology (Oxford) 2004;43:858-62.

[30] Tarnopolsky MA, Bourgeois JM, Snow R, Keys S, Roy BD, Kwiecien JM, et al. Histological assessment of intermediate- and long-term creatine monohydrate supplementation in mice and rats. Am J Physiol Regul Integr Comp Physiol 2003;285:R762-9.

[31] Kyrlagkitsis I, Portmann B, Smith H, O'Grady J, Cramp ME. Liver histology and progression of fibrosis in individuals with chronic hepatitis $C$ and persistently normal ALT. Am J Gastroenterol 2003;98:1588-93.

[32] Fontenot JD, Rasmussen JP, Williams LM, Dooley JL, Farr AG, Rudensky AY. Regulatory $T$ cell lineage specification by the forkhead transcription factor foxp3. Immunity 2005;22:329-41.

[33] Marie JC, Liggitt D, Rudensky AY. Cellular mechanisms of fatal early-onset autoimmunity in mice with the $\mathrm{T}$ cell-specific targeting of transforming growth factor- $\beta$ receptor. Immunity 2006;25:441-54.

[34] Fedeli M, Napolitano A, Wong MP, Marcais A, de Lalla C, Colucci F, et al. Dicerdependent microRNA pathway controls invariant NKT cell development. J Immunol 2009;183:2506-12.
[35] Asano M, Toda M, Sakaguchi N, Sakaguchi S. Autoimmune disease as a consequence of developmental abnormality of a T cell subpopulation. J Exp Med 1996;184:387-96.

[36] Powrie F, Leach MW, Mauze S, Menon S, Caddle LB, Coffman RL. Inhibition of Th1 responses prevents inflammatory bowel disease in scid mice reconstituted with CD45RBhi CD4+ T cells. Immunity 1994;1:553-62.

[37] Powrie F, Correa-Oliveira R, Mauze S, Coffman RL. Regulatory interactions between CD45RBhigh and CD45RBlow CD4 + T cells are important for the balance between protective and pathogenic cell-mediated immunity. J Exp Med 1994;179:589-600.

[38] Huber S, Gagliani N, Esplugues E, O'Connor Jr W, Huber FJ, Chaudhry A, et al. Th17 cells express interleukin-10 receptor and are controlled by Foxp3 and Foxp3 + regulatory CD4 $+\mathrm{T}$ cells in an interleukin-10-dependent manner. Immunity 2011;34:554-65.

[39] Dai Y, Huang YS, Tang M, Lv TY, Hu CX, Tan YH, et al. Microarray analysis of microRNA expression in peripheral blood cells of systemic lupus erythematosus patients. Lupus 2007;16:939-46.

[40] Pauley KM, Satoh M, Chan AL, Bubb MR, Reeves WH, Chan EK. Upregulated miR-146a expression in peripheral blood mononuclear cells from rheumatoid arthritis patients. Arthritis Res Ther 2008;10:R101.

[41] Wiesen JL, Tomasi TB. Dicer is regulated by cellular stresses and interferons. Mol Immunol 2009;46:1222-8. 